\title{
Polynomial Inequalities in Regions Bounded by Piecewise Asymptotically Conformal Curve with Nonzero Angles in the Bergman Space
}

\author{
G. A. ABDULLAYEV ${ }^{1 *}$, F. G. ABDULLAYEV ${ }^{1,2 * *}$ and A. TAYLAKOVA ${ }^{2}$ \\ ${ }^{1}$ Mersin University, Mersin, Turkey \\ ${ }^{2}$ Kyrgyz-Turkish Manas University, Bishkek, Kyrgyzstan \\ Email: ${ }^{*}$ gulnareabdullah@mersin.edu.tr, ${ }^{* *}$ fabdul@mersin.edu.tr, ${ }^{* *}$ fahreddinabdullayev@gmail.com
}

\begin{abstract}
We continue the study of estimates of algebraic polynomials in regions bounded by a piecewise asymptotically conformal curve with interior non-zero angles in the weighted Bergman space.
\end{abstract}

Keywords: Algebraic polynomials, Conformal mapping, Asymptotically conformal curve.

Prim.30A10, 30C10, Sec.41A17

\section{Introduction and Main Results}

Let $G \subset \mathbb{C}$ be a finite region, with $0 \in G$, bounded by a Jordan curve $L:=\partial G, \Omega:=\operatorname{ext} L:=\overline{\mathbb{C}} \backslash \bar{G}$, where $\overline{\mathbb{C}}:=\mathbb{C} \cup\{\infty\}, \Delta:=\{w:|w|>1\}$ and let $\wp_{n}$ denote the class of arbitrary algebraic polynomials $P_{n}(z)$ of degree at most $n \in \mathbb{N}$. Let $w=\Phi(z)$ be the univalent conformal mapping of $\Omega$ onto the $\Delta$ normalized by $\Phi(\infty)=\infty, \lim _{z \rightarrow \infty} \frac{\Phi(z)}{z}>0$, and $\Psi:=\Phi^{-1}$. For $t \geq 1, z \in \mathbb{C}$, we set:

$$
L_{t}:=\{z:|\Phi(z)|=t\} \quad\left(L_{1} \equiv L\right), G_{t}:=\operatorname{int} L_{t}, \Omega_{t}:=\operatorname{ext} L_{t} .
$$

Let $\left\{z_{j}\right\}_{j=1}^{m}$ be a fixed system of distinct points on curve $L$, located in the positive direction. For some fixed $R_{0}, 1<R_{0}<\infty$, and $z \in G_{R_{0}}$, consider a so-called generalized Jacobi weight function $h(z)$ being defined as follows:

$$
h(z):=h_{0}(z) \prod_{j=1}^{m}\left|z-z_{j}\right|^{\gamma_{j}}, \quad z \in G_{R_{0}},
$$

where $\gamma_{j}>-2$, for all $j=1,2, \ldots, m$, and the function $h_{0}$ is uniformly separated from zero in $G_{R_{0}}$, i.e. there exists a constant $c_{0}:=c_{0}\left(G_{R_{0}}\right)>0$ such that, for all $z \in G_{R_{0}}$

$$
h_{0}(z) \geq c_{0}>0 .
$$

For any $p>0$ and for Jordan region $G$, let's define:

$$
\begin{aligned}
\left\|P_{n}\right\|_{p} & :=\left\|P_{n}\right\|_{A_{p}(h, G)}:=\left(\iint_{G} h(z)\left|P_{n}(z)\right|^{p} d \sigma_{z}\right)^{1 / p}<\infty, 0<p<\infty ; \\
\left\|P_{n}\right\|_{\infty}: & :\left\|P_{n}\right\|_{A_{\infty}(1, G)}:=\left\|P_{n}\right\|_{C(\bar{G})}, p=\infty,
\end{aligned}
$$

where $\sigma_{z}$ is the two-dimensional Lebesgue measure.

In this work, we continue the study of the following Nikolskii-type inequality:

$$
\left\|P_{n}\right\|_{\infty} \leq c_{1} \lambda_{n}(G, h, p)\left\|P_{n}\right\|_{p},
$$

\footnotetext{
** Corresponding Author
} 
where $c_{1}=c_{1}(G, h, p)>0$ is a constant independent of $n$ and $P_{n}$, and $\lambda_{n}(G, h, p) \rightarrow \infty, n \rightarrow \infty$, depending on the geometrical properties of region $G$, weight function $h$ and of $p$. The estimate of (1.3)type for some $(G, p, h)$ was investigated in [27, pp.122-133], [17], [26, Sect.5.3], [32], [15], [2]-[8] (see, also, references therein) and others. Further, analogous of (1.3) for some regions and the weight function $h(z)$ were obtained: in [8] for $p>1$ and for regions bounded by piecewise Dini-smooth boundary without cusps; in [11] for $p>0$ and for regions bounded by quasiconformal curve; in [7] for $p>1$ and for regions bounded by piecewise smooth curve without cusps; in [10] for $p>0$ and for regions bounded by asymptotically conformal curve; in [16] for $p>0$ and for regions bounded by piesewise smooth curves with interior (zero or nonzero) angles, in [12] for $p>0$ and for regions bounded by piecewise asymptotically conformal curve having cusps and others.

In this work, we investigate similar problems for $z \in \bar{G}$ in regions bounded by piecewise asymptotically conformal curves having interior nonzero zero angles and for weight function $h(z)$, defined in (1.1) and for $p>0$.

Now, we begin to give some definitions and notations.

Following [24, p.97], [28], the Jordan curve (or arc) $L$ is called $K$-quasiconformal $(K \geq 1)$, if there is a $K$-quasiconformal mapping $f$ of the region $D \supset L$ such that $f(L)$ is a circle (or line segment).

Let $S$ be a Jordan curve and $z=z(s), s \in[0,|S|],|S|:=$ mes $S$, denote the natural representation of $S$. Let $z_{1}, z_{2} \in S$ be an arbitrary points and $S\left(z_{1}, z_{2}\right) \subset S$ denotes the subarc of $S$ of shorter diameter with endpoints $z_{1}$ and $z_{2}$. The curve $S$ is a quasicircle if and only if the quantity

$$
\sup _{z_{1}, z_{2} \in l ; z \in l\left(z_{1}, z_{2}\right)} \frac{\left|z_{1}-z\right|+\left|z-z_{2}\right|}{\left|z_{1}-z_{2}\right|}
$$

is bounded. Following to Lesley [25], the curve $S$ to be said "c-quasiconformal", if the quantity (1.4) bounded by positive constant $c$, independent from points $z_{1}, z_{2}$ and $z$. At the literature it is possible to find various functional definitions of the quasiconformal curves (see, for example, [29, pp.286-294], [24, p.105], [13, p.81], [30, p.107]).

The Jordan curve $S$ is called asymptotically conformal [19], [30], if

$$
\sup _{z_{1}, z_{2} \in S ; z \in S\left(z_{1}, z_{2}\right)} \frac{\left|z_{1}-z\right|+\left|z-z_{2}\right|}{\left|z_{1}-z_{2}\right|} \rightarrow 1, \quad\left|z_{1}-z_{2}\right| \rightarrow 0 .
$$

We will denote this class as $A C$, and will write $G \in A C$, if $L:=\partial G \in A C$.

The asymptotically conformal curves occupy a special place in the problems of the geometric theory of functions of a complex variable. These curves in various problems have been studied by Anderson, Becker and Lesley [14], Dyn'kin [20], Pommerenke, Warschawski [31], Gutlyanskii, Ryazanov [21], [22], [23] and others. According to the geometric criteria of quasiconformality of the curves ([13, p.81], [30, p.107]), every asymptotically conformal curve is a quasicircle. Every smooth curve is asymptotically conformal but corners are not allowed. It is well known that quasicircles can be non-rectifiable (see, for example, [18], [24, p.104]). The same is true for asymptotically conformal curves.

A Jordan arc $\ell$ is called asymptotically conformal arc, when $\ell$ is a part of some asymptotically conformal curve.

Now, we define a new class of regions bounded by piecewise asymptotically conformal curve having exterior nonzero "angles" at the connecting points of boundary arcs.

Throughout this work, we will assume that $p>0$ and the constants $c, c_{0}, c_{1}, c_{2}, \ldots$ are positive and constants $\varepsilon_{0}, \varepsilon_{1}, \varepsilon_{2}, \ldots$ are sufficiently small positive (generally, are different in different relations), which depends on $G$ in general and, on parameters inessential for the argument, otherwise, the dependence will be explicitly stated. Also note that, for any $k \geq 0$ and $m>k$, notation $j=\overline{k, m}$ denotes $j=k, k+1, \ldots, m$.

Now, let's introduce "special angles" on $L$.

Definition 1.1. We say that a Jordan region $G \in P A C\left(\nu_{1}, \ldots, \nu_{m}\right), 0<\nu_{j}<2, j=\overline{1, m}$, if $L=\partial G$ consists of the union of finite asymptotically conformal arcs $\left\{L_{j}\right\}_{j=1}^{m}$, connected at the points $\left\{z_{j}\right\}_{j=0}^{m} \in$ $L$ such that in $z_{0^{-}} L$ locally asymptotically conformal and for any $z_{j} \in L, j=\overline{1, m}$, where two arcs $L_{j-1}$ and $L_{j}$ meet, there exist $r_{j}:=r_{j}\left(L, z_{j}\right)>0$ and $\nu_{j}:=\nu_{j}\left(L, z_{j}\right), 0<\nu_{j}<2$, such that for some $0 \leq \theta_{0}<2$ a closed maximal circular sector $S\left(z_{j} ; r_{j}, \nu_{j}\right):=\left\{\zeta: \zeta=z_{j}+r_{j} e^{i \theta \pi}, \theta_{0}<\theta<\theta_{0}+\nu_{j}\right\}$ of radius $r_{j}$ and opening $\nu_{j} \pi$ lies in $\bar{G}=\overline{\text { intL }}$ with vetrex at $z_{j}$. 
Clearly, that $P A C\left(\nu_{1}\right) \subset P A C\left(\nu_{2}\right)$, if $\nu_{2} \geq \nu_{1}$.

Definition 1.2. We say that a Jordan region $G \in P A C(\nu)$, if $G \in P A C\left(\nu_{1}, \ldots, \nu_{m}\right), 0<\nu_{j}<2, j=\overline{1, m}$, where $\nu=\min \left\{\nu_{j}: 0<\nu_{j}<2, j=\overline{1, m}\right\}$.

It is clear from Definition $1.1(1.2)$, that each region $G \in P A C\left(\nu_{1}, \ldots, \nu_{m}\right), 0<\nu_{1}, \ldots, \nu_{m}<2$, $(G \in P A C(\nu))$ may have "singularity" at the boundary points $\left\{z_{i}\right\}_{i=1}^{m} \in L$. If it does not have such "singularity" ( in this case we put $\nu_{i}=1, i=\overline{1, m}$ ), then it is written as $G \in A C$.

Throughout this work, we will assume that the points $\left\{z_{i}\right\}_{i=1}^{m} \in L$ defined in (1.1) and $\left\{\zeta_{i}\right\}_{i=1}^{m} \in L$ defined in Definition 1.1 (1.2) coincide. Without the loss of generality, we also will assume that the points $\left\{z_{i}\right\}_{i=1}^{m}$ are ordered in the positive direction on the curve $L$.

We state our new results. Assume that the curve $L$ have "singularity" on the boundary points $\left\{z_{i}\right\}_{i=1}^{m}$, i.e., $\nu_{i}<1$, for all $i=\overline{1, m}$, and the weight function $h$ have "singularity" at the same points, i.e., $\gamma_{i} \neq 0$ for some $i=\overline{1, m}$. In this case, we have the following:

Theorem 1.1. Let $p>0$. Suppose that $G \in P A C\left(\nu_{1}, \ldots, \nu_{m}\right)$ for some $0<\nu_{1}, \ldots, \nu_{m}<1 ; h(z)$ defined as in (1.1). Then, for any $P_{n} \in \wp_{n}, n \in \mathbb{N}$, and arbitrarily small $\varepsilon>0$, there exists $c_{1}=c_{1}\left(G, p, \gamma_{j}\right)>0$ such that

$$
\left\|P_{n}\right\|_{\infty} \leq c_{1}(n+1)^{\frac{2+\widetilde{\gamma}}{p}(2-\widetilde{\nu})+\varepsilon}\left\|P_{n}\right\|_{p}
$$

where $\widetilde{\gamma}:=\max \left\{0, \gamma_{i}\right\}$ and $\widetilde{\nu}:=\min \left\{\nu_{i}\right\}, i=\overline{1, m}$.

Theorem 1.2. Let $p>0$. Suppose that $G \in P A C\left(\nu_{1}, \ldots, \nu_{m}\right)$ for some $0<\nu_{1}, \ldots, \nu_{m}<1 ; h(z)$ defined as in (1.1). Then, for any $P_{n} \in \wp_{n}, n \in \mathbb{N}$, and arbitrarily small $\varepsilon>0$, there exists $c_{2}=c_{2}\left(G, p, \gamma_{j}\right)>0$ such that

$$
\left|P_{n}\left(z_{j}\right)\right| \leq c_{2} \mu_{n}\left\|P_{n}\right\|_{p},
$$

where

$$
\mu_{n}:=\left\{\begin{array}{cc}
n \frac{\left(2+\gamma_{j}\right)\left(2-\nu_{j}\right)}{p}+\varepsilon, & \text { if } \gamma_{j}>\frac{1}{2-\nu_{j}}-2-\varepsilon, \\
(n \ln n)^{\frac{1}{p}}, & \text { if } \gamma_{j}=\frac{1}{2-\nu_{j}}-2-\varepsilon, \\
n^{\frac{1}{p}}, & \text { if }-2<\gamma_{j}<\frac{1}{2-\nu_{j}}-2-\varepsilon
\end{array}\right.
$$

The sharpness of the estimations (1.6) and (1.7) can be discussed by comparing them with the following result:

Remark 1.1. ([9, Theorem 1.15], [2]) For any $n \in \mathbb{N}$ there exists a polynomials $Q_{n}^{*}, T_{n}^{*} \in \wp_{n}$ such that for unit disk $B$ and weight function $h^{*}(z)=\left|z-z_{1}\right|^{2}$ the following is true:

$$
\begin{aligned}
\left|Q_{n}^{*}(z)\right| & \geq c_{6} n\left\|Q_{n}^{*}\right\|_{A_{2}(B)}, \quad \text { for all } z \in \bar{B} ; \\
\left|T_{n}^{*}\left(z_{1}\right)\right| & \geq c_{7} n^{2}\left\|T_{n}^{*}\right\|_{A_{2}\left(h^{*}, B\right)} ;
\end{aligned}
$$

\section{Some Auxiliary Results}

Throughout this work, for the nonnegative functions $a>0$ and $b>0$, we shall use the notations " $a \preceq b$ " (order inequality), if $a \leq c b$ and " $a \asymp b$ " are equivalent to $c_{1} a \leq b \leq c_{2} a$ for some constants $c, c_{1}, c_{2}$ (independent of $a$ and $b$ ), respectively.

Lemma 2.1. [1] Let $L$ be a $K$-quasiconformal curve, $z_{1} \in L, z_{2}, z_{3} \in \Omega \cap\left\{z:\left|z-z_{1}\right| \preceq d\left(z_{1}, L_{r_{0}}\right)\right\}$; $w_{j}=\Phi\left(z_{j}\right), j=1,2,3$. Then

a) The statements $\left|z_{1}-z_{2}\right| \preceq\left|z_{1}-z_{3}\right|$ and $\left|w_{1}-w_{2}\right| \preceq\left|w_{1}-w_{3}\right|$ are equivalent.

So are $\left|z_{1}-z_{2}\right| \asymp\left|z_{1}-z_{3}\right|$ and $\left|w_{1}-w_{2}\right| \asymp\left|w_{1}-w_{3}\right|$;

b) If $\left|z_{1}-z_{2}\right| \preceq\left|z_{1}-z_{3}\right|$, then

$$
\left|\frac{w_{1}-w_{3}}{w_{1}-w_{2}}\right|^{K^{-2}} \preceq\left|\frac{z_{1}-z_{3}}{z_{1}-z_{2}}\right| \preceq\left|\frac{w_{1}-w_{3}}{w_{1}-w_{2}}\right|^{K^{2}},
$$

where $0<r_{0}<1, R_{0}:=r_{0}^{-1}$ are constants, depending on $G$. 
Lemma 2.2. [25, p.342] Let $L$ be an asymptotically conformal curve. Then, $\Phi$ and $\Psi$ are Lipa for all $\alpha<1$ in $\bar{\Omega}$ and $\bar{\Delta}$, correspondingly.

Let $\left\{z_{j}\right\}_{j=1}^{m}$ be a fixed system of the points on $L$ and the weight function $h(z)$ be defined as in (1.1). Lemma 2.3. [5] Let $L$ be a $K$-quasiconformal curve; $h(z)$ is defined in (1.1). Then, for arbitrary $P_{n}(z) \in \wp_{n}$, any $R>1$ and $n=1,2, \ldots$, we have

$$
\left\|P_{n}\right\|_{A_{p}\left(h, G_{R}\right)} \preceq \widetilde{R}^{n+\frac{1}{p}}\left\|P_{n}\right\|_{A_{p}(h, G)}, p>0,
$$

where $\widetilde{R}=1+c(R-1)$ and $c$ is independent from $n$ and $R$.

\section{Proof of Theorems}

\subsection{Proof of Theorem 1.1}

Proof. Suppose that $G \in P A C\left(\nu_{1}, \nu_{2}\right)$ for some $0<\nu_{1}, \nu_{2}<1$ and $h(z)$ is defined as in (1.1). Let $\left\{\xi_{j}\right\}, 1 \leq j \leq m \leq n$, be the zeros (if any exist) of $P_{n}(z)$ lying on $\Omega$. Let's define the function Blaschke with respect to the zeros $\left\{\xi_{j}\right\}$ of the polynomial $P_{n}(z)$ :

$$
\widetilde{B}_{j}(z):=\frac{\Phi(z)-\Phi\left(\xi_{j}\right)}{1-\overline{\Phi\left(\xi_{j}\right)} \Phi(z)}, z \in \Omega,
$$

and let

$$
B_{m}(z):=\prod_{j=1}^{m} \widetilde{B}_{j}(z), z \in \Omega .
$$

It is easy that the

$$
B_{m}\left(\xi_{j}\right)=0, \quad\left|B_{m}(z)\right| \equiv 1, z \in L ;\left|B_{m}(z)\right|<1, z \in \Omega .
$$

Then, for each $\varepsilon_{1}, 0<\varepsilon_{1}<1$, there exists a circle $\left\{w:|w|=R_{1}:=1+\varepsilon_{2}, 0<\varepsilon_{2}<\frac{\varepsilon_{1}}{n}\right\}$ such that for any $j=1,2$, the following holds:

$$
\left|\widetilde{B}_{j}(\zeta)\right|>1-\varepsilon_{2}, \zeta \in L_{R_{1}}
$$

So, from (3.2), we get:

$$
\left|B_{m}(\zeta)\right|>\left(1-\varepsilon_{2}\right)^{m} \succeq 1, \zeta \in L_{R_{1}} .
$$

For any $p>0$ and $z \in \Omega$ let us set:

$$
Q_{n, p}(z):=\left[\frac{P_{n}(z)}{B_{m}(z) \Phi^{n+1}(z)}\right]^{p / 2} .
$$

The function $Q_{n, p}(z)$ is analytic in $\Omega$, continuous on $\bar{\Omega}, Q_{n, p}(\infty)=0$ and does not have zeros in $\Omega$. We take an arbitrary continuous branch of the $Q_{n, p}(z)$ and for this branch, we maintain the same designation. According to Cauchy integral representation for the unbounded region $\Omega$, we have:

$$
Q_{n, p}(z)=-\frac{1}{2 \pi i} \int_{L_{R_{1}}} Q_{n, p}(\zeta) \frac{d \zeta}{\zeta-z}, z \in \Omega_{R_{1}} .
$$

According to $(3.1)-(3.5)$, we have:

$$
\begin{aligned}
\left|P_{n}(z)\right|^{p / 2} & =\frac{\left|B_{m}(z) \Phi^{n+1}(z)\right|^{\frac{p}{2}}}{2 \pi d\left(z, L_{R_{1}}\right)} \int_{L_{R_{1}}}\left|\frac{P_{n}(\zeta)}{B_{m}(\zeta) \Phi^{n+1}(\zeta)}\right|^{p / 2}|d \zeta| \\
& \preceq\left|\Phi^{n+1}(z)\right|^{\frac{p}{2}} \int_{L_{R_{1}}}\left|P_{n}(\zeta)\right|^{p / 2} \frac{|d \zeta|}{|\zeta-z|} .
\end{aligned}
$$


Multiplying the numerator and the denominator of the last integrand by $h^{1 / 2}(\zeta)$, replacing the variable $w=\Phi(z)$ and applying the Hölder inequality, we obtain:

$$
\begin{aligned}
&\left(\int_{L_{R_{1}}}\left|P_{n}(\zeta)\right|^{\frac{p}{2}}|d \zeta|\right)^{2} \leq \int_{|t|=R_{1}} h(\Psi(t))\left|P_{n}(\Psi(t))\right|^{p}\left|\Psi^{\prime}(t)\right|^{2}|d t| \cdot \int_{|t|=R_{1}} \frac{|d t|}{h(\Psi(t))|\Psi(t)-\Psi(w)|^{2}} \\
& \quad \leq \int_{|t|=R_{1}} h(\Psi(t))\left|P_{n}(\Psi(t))\right|^{p}\left|\Psi^{\prime}(t)\right|^{2}|d t| \cdot \int_{|t|=R_{1}} \frac{|d t|}{h(\Psi(t))|\Psi(t)-\Psi(w)|^{2}} \\
&=\int_{|t|=R_{1}}\left|f_{n, p}(t)\right|^{p}|d t| \cdot \int_{|t|=R_{1}} \frac{|d t|}{h(\Psi(t))|\Psi(t)-\Psi(w)|^{2}}=: A_{n} \cdot D_{n}(w),
\end{aligned}
$$

where $f_{n, p}(t):=h^{\frac{1}{p}}(\Psi(t)) P_{n}(\Psi(t))\left(\Psi^{\prime}(t)\right)^{\frac{2}{p}},|t|=R_{1}$.

For the estimate integral $A_{n}$, we divide the circle $|t|=R_{1}$ into $n$ equal parts $\delta_{n}$ with $m e s \delta_{n}=\frac{2 \pi R_{1}}{n}$ and by applying the mean value theorem, we get:

$$
\begin{aligned}
& A_{n}:=\int_{|t|=R_{1}}\left|f_{n, p}(t)\right|^{p}|d t| \\
= & \sum_{k=1}^{n} \int_{\delta_{k}}\left|f_{n, p}(t)\right|^{p}|d t|=\sum_{k=1}^{n}\left|f_{n, p}\left(t_{k}^{\prime}\right)\right|^{p} \operatorname{mes}_{k}, \quad t_{k}^{\prime} \in \delta_{k} .
\end{aligned}
$$

On the other hand, by applying mean value estimation

$$
\left|f_{n, p}\left(t_{k}^{\prime}\right)\right|^{p} \leq \frac{1}{\pi\left(\left|t_{k}^{\prime}\right|-1\right)^{2}} \iint_{\left|\xi-t_{k}^{\prime}\right|<\left|t_{k}^{\prime}\right|-1}\left|f_{n, p}(\xi)\right|^{p} d \sigma_{\xi},
$$

we obtain:

$$
\left(A_{n}\right)^{2} \preceq \sum_{k=1}^{n} \frac{m e s \delta_{k}}{\pi\left(\left|t_{k}^{\prime}\right|-1\right)^{2}} \underset{\left|\xi-t_{k}^{\prime}\right|<\left|t_{k}^{\prime}\right|-1}{\int}\left|f_{n, p}(\xi)\right|^{p} d \sigma_{\xi}, t_{k}^{\prime} \in \delta_{k} .
$$

By taking into account, at most two of the discs with center $t_{k}^{\prime}$ are intersecting, we have:

$$
A_{n} \preceq \frac{m e s \delta_{1}}{\left(\left|t_{1}^{\prime}\right|-1\right)^{2}} \iint_{1<|\xi|<R}\left|f_{n, p}(\xi)\right|^{p} d \sigma_{\xi} \preceq n \cdot \iint_{1<|\xi|<R}\left|f_{n, p}(\xi)\right|^{p} d \sigma_{\xi} .
$$

According to Lemma 2.3, for $A_{n}$ we get:

$$
A_{n} \preceq n \iint_{G_{R} \backslash G} h(\zeta)\left|P_{n}(\zeta)\right|^{p} d \sigma_{\zeta} \preceq n \cdot\left\|P_{n}\right\|_{p}^{p} .
$$

To estimate the integral $D_{n}(w)$, denoted by $w_{j}:=\Phi\left(z_{j}\right), \varphi_{j}:=\arg w_{j}$, for any fixed $\rho>1$, we introduce:

$$
\begin{gathered}
\Delta_{1}(\rho):=\left\{t=r e^{i \theta}: r>\rho, \frac{\varphi_{0}+\varphi_{1}}{2} \leq \theta<\frac{\varphi_{1}+\varphi_{2}}{2}\right\}, \\
\Delta_{2}(\rho):=\left\{t=r e^{i \theta}: r>\rho, \frac{\varphi_{1}+\varphi_{2}}{2} \leq \theta<\frac{\varphi_{1}+\varphi_{0}}{2}\right\} ; \\
\Delta_{j}:=\Delta_{j}(1), \Omega^{j}:=\Psi\left(\Delta_{j}\right), \Omega_{\rho}^{j}:=\Psi\left(\Delta_{j}(\rho)\right) ; \\
L^{j}:=L \cap \bar{\Omega}^{j}, L_{\rho}^{j}:=L_{\rho} \cap \bar{\Omega}_{\rho}^{j}, j=1,2 ; L=L^{1} \cup L^{1}, L_{\rho}=L_{\rho}^{1} \cup L_{\rho}^{2} .
\end{gathered}
$$


Under these notations, from (3.8) for the $D_{n}(w)$, we get:

$$
\begin{gathered}
D_{n}(w)=\int_{|t|=R_{1}} \frac{|d t|}{h(\Psi(t))|\Psi(t)-\Psi(w)|^{2}} \\
\preceq \sum_{j=1}^{2} \int_{\Phi\left(L_{R_{1}}^{j}\right)} \frac{|d t|}{\prod_{j=1}^{2}\left|\Psi(t)-\Psi\left(w_{j}\right)\right|^{\gamma_{j}}|\Psi(t)-\Psi(w)|^{2}} \\
\asymp \sum_{j=1}^{2} \int_{\Phi\left(L_{R_{1}}^{j}\right)} \frac{|d t|}{\left|\Psi(t)-\Psi\left(w_{j}\right)\right|^{\gamma_{j}}|\Psi(t)-\Psi(w)|^{2}}=: \sum_{j=1}^{2} D_{n, j}(w),
\end{gathered}
$$

since the points $\left\{z_{j}\right\}_{j=1}^{2} \in L$ are distinct. So, we need to evaluate the $D_{n, j}(w)$. For this, we take $z \in L_{R}$ and introduce the notations:

$$
\Phi\left(L_{R_{1}}\right)=\Phi\left(\bigcup_{j=1}^{2} L_{R_{1}}^{j}\right)=\bigcup_{j=1}^{2} \Phi\left(L_{R_{1}}^{j}\right)=\bigcup_{j=1}^{2} \bigcup_{i=1}^{2} K_{i}^{j}\left(R_{1}\right),
$$

where

$$
\begin{aligned}
& K_{1}^{j}\left(R_{1}\right):=\left\{t \in \Phi\left(L_{R_{1}}^{j}\right):\left|t-w_{j}\right|<c_{1}\right\} \\
& K_{2}^{j}\left(R_{1}\right):=\Phi\left(L_{R_{1}}^{j}\right) \backslash K_{1}^{j}\left(R_{1}\right), j=1,2 .
\end{aligned}
$$

Analogously,

$$
\Phi\left(L_{R}\right)=\Phi\left(\bigcup_{j=1}^{2} L_{R}^{j}\right)=\bigcup_{j=1}^{2} \Phi\left(L_{R}^{j}\right)=\bigcup_{j=1}^{2} \bigcup_{i=1}^{2} K_{i}^{j}(R)
$$

where

$$
\begin{aligned}
& K_{1}^{j}(R):=\left\{t \in \Phi\left(L_{R}^{j}\right):\left|\tau-w_{j}\right|<2 c_{1}\right\} \\
& K_{2}^{j}(R):=\Phi\left(L_{R}^{j}\right) \backslash K_{1}^{j}(R), j=1,2 .
\end{aligned}
$$

Then, after these definitions, taking arbitrary fixed $w=\Phi(z) \in \Phi\left(L_{R}\right)$, the quantity $D_{n, j}(w)$ can be written as follows:

$$
D_{n, j}(w)=\sum_{i=1}^{2} \int_{K_{i}^{j}\left(R_{1}\right)} \frac{|d t|}{\left|\Psi(t)-\Psi\left(w_{j}\right)\right|^{\gamma_{j}}|\Psi(t)-\Psi(w)|^{2}}=: \sum_{i=1}^{2} D_{n, j}^{i}(w)
$$

The quantity $D_{n, j}^{i}(w)$ we shall estimate for each $i=1,2$ and $j=1,2$ in cases separately, depending of location of the $w \in \Phi\left(L_{R}\right)$. Let $\varepsilon>0$ arbitrary small fixed number.

Case 1. Let $w \in \Phi\left(L_{R}^{1}\right)$.

According to the above notations, we will make evaluations for case $w \in K_{i}^{1}(R)$ for each $i=1,2,3$.

1.1) Let $w \in K_{1}^{1}(R)$. In this case, we will estimate the quantity

$$
D_{n, 1}(w)=\sum_{i=1}^{2} \int_{K_{i}^{1}\left(R_{1}\right)} \frac{|d t|}{\left|\Psi(t)-\Psi\left(w_{1}\right)\right|^{\gamma_{1}}|\Psi(t)-\Psi(w)|^{2}}=: \sum_{i=1}^{2} D_{n, 1}^{i}(w)
$$

for $\gamma_{1} \geq 0$ and $\gamma_{1}<0$ separately.

For each $i=1,2$ and $j=1,2$ we put: $K_{i, 1}^{j}\left(R_{1}\right):=\left\{t \in \Phi\left(L_{R_{1}}^{j}\right):\left|t-w_{j}\right| \geq|t-w|\right\}, \quad K_{i, 2}^{j}\left(R_{1}\right):=$ $K_{i}^{j}\left(R_{1}\right) \backslash K_{i, 1}^{j}\left(R_{1}\right)$. 
1.1.1) If $\gamma_{1} \geq 0$, then

$$
\begin{aligned}
D_{n, 1}^{1}(w) & =\int_{K_{1}^{1}\left(R_{1}\right)} \frac{|d t|}{\left|\Psi(t)-\Psi\left(w_{1}\right)\right|^{\gamma_{1}}|\Psi(t)-\Psi(w)|^{2}} \\
& =\int_{K_{1,1}^{1}\left(R_{1}\right)} \frac{|d t|}{|\Psi(t)-\Psi(w)|^{2+\gamma_{1}}}+\int_{K_{1,2}^{1}\left(R_{1}\right)} \frac{|d t|}{\left|\Psi(t)-\Psi\left(w_{1}\right)\right|^{2+\gamma_{1}}} \\
& =: D_{n, 1}^{1,1}(w)+D_{n, 1}^{1,2}(w) .
\end{aligned}
$$

Since $G \in P A C\left(\nu_{1}, \nu_{2}\right)$ for some $0<\nu_{1}, \nu_{2}<1$, according to [25], $\psi \in \operatorname{Lip} \nu_{i}$ and $\Phi \in \operatorname{Lip} \frac{1}{2-\nu_{i}}, i=1,2$, in a some fixed neighborhood of point $z_{j}$. Therefore, we get:

$$
D_{n, 1}^{1,1}(w) \preceq \int_{K_{1,1}^{1}\left(R_{1}\right)} \frac{|d t|}{|t-w|^{\left(2+\gamma_{1}\right)\left(2-\nu_{1}\right)}} \preceq n^{\left(2+\gamma_{1}\right)\left(2-\nu_{1}\right)-1},
$$

and

$$
D_{n, 1}^{1,2}(w) \preceq \int_{K_{1,2}^{1}\left(R_{1}\right)} \frac{|d t|}{\left|t-w_{1}\right|^{\left(2+\gamma_{1}\right)\left(2-\nu_{1}\right)}} \preceq n^{\left(2+\gamma_{1}\right)\left(2-\nu_{1}\right)-1},
$$

If $\gamma_{1}<0$, then

$$
\begin{aligned}
& D_{n, 1}^{1}(w)=\int_{K_{1}^{1}\left(R_{1}\right)} \frac{\left|\Psi(t)-\Psi\left(w_{1}\right)\right|^{\left(-\gamma_{1}\right)}|d t|}{|\Psi(t)-\Psi(w)|^{2}} \\
& \preceq \int_{K_{1}^{1}\left(R_{1}\right)} \frac{|d t|}{|t-w|^{2\left(2-\nu_{1}\right)}} \preceq \int_{K_{1}^{1}\left(R_{1}\right)} \frac{|d t|}{|t-w|^{2\left(2-\nu_{1}\right)}} \\
& \preceq n^{2\left(2-\nu_{1}\right)-1} .
\end{aligned}
$$

1.1.2) If $\gamma_{1} \geq 0$, then

$$
\begin{aligned}
D_{n, 1}^{2}(w) & =\int_{K_{2}^{1}\left(R_{1}\right)} \frac{|d t|}{\left|\Psi(t)-\Psi\left(w_{1}\right)\right|^{\gamma_{1}}|\Psi(t)-\Psi(w)|^{2}} \\
& =\int_{K_{2,1}^{1}\left(R_{1}\right)} \frac{|d t|}{|\Psi(t)-\Psi(w)|^{2+\gamma_{1}}}+\int_{K_{2,2}^{1}\left(R_{1}\right)} \frac{|d t|}{\left|\Psi(t)-\Psi\left(w_{1}\right)\right|^{2+\gamma_{1}}} \\
& =: D_{n, 1}^{2,1}(w)+D_{n, 1}^{2,2}(w) .
\end{aligned}
$$

and, so from Lemma 2.1 and 2.2, we get:

$$
D_{n, 1}^{2,1}(w) \preceq \int_{K_{2,1}^{1}\left(R_{1}\right)} \frac{|d t|}{|t-w|^{\left(2+\gamma_{1}\right)\left(2-\nu_{1}\right)}} \preceq n^{\left(2+\gamma_{1}\right)\left(2-\nu_{1}\right)-1},
$$

and

$$
D_{n, 1}^{2,2}(w) \preceq 1 .
$$

Therefore, from (3.19)-(3.21) for $\gamma_{1} \geq 0$, we have:

$$
D_{n, 1}^{2}(w) \preceq n^{\left(2+\gamma_{1}\right)\left(2-\nu_{1}\right)-1} .
$$

For $\gamma_{1}<0$ from (3.14), we have:

$$
D_{n, 1}^{2}(w)=\int_{K_{2}^{1}\left(R_{1}\right)} \frac{\left|\Psi(t)-\Psi\left(w_{1}\right)\right|^{\left(-\gamma_{1}\right)}|d t|}{|\Psi(t)-\Psi(w)|^{2}}
$$




$$
\preceq \int_{K_{2}^{1}\left(R_{1}\right)} \frac{|d t|}{|t-w|^{2(1+\varepsilon)}} \preceq n^{1+\varepsilon}, \forall \varepsilon>0 .
$$

1.2) Let $w \in K_{2}^{1}(R)$.

1.2.1) For any $\gamma_{1}>-2$

$$
\begin{aligned}
D_{n, 1}^{1}(w) & =\int_{K_{1,1}^{1}\left(R_{1}\right)} \frac{|d t|}{|\Psi(t)-\Psi(w)|^{2+\gamma_{1}}}+\int_{K_{1,2}^{1}\left(R_{1}\right)} \frac{|d t|}{\left|\Psi(t)-\Psi\left(w_{1}\right)\right|^{2+\gamma_{1}}} \\
& =: D_{n, 1}^{1,1}(w)+D_{n, 1}^{1,2}(w),
\end{aligned}
$$

and so, according to Lemmas 2.1 and 2.2, we obtain:

$$
D_{n, 1}^{1,1}(w) \preceq \int_{K_{1,1}^{1}\left(R_{1}\right)} \frac{|d t|}{|\Psi(t)-\Psi(w)|^{2+\gamma_{1}}} \preceq 1,
$$

and

$$
D_{n, 1}^{1,2}(w) \preceq \int_{K_{1,2}^{1}\left(R_{1}\right)} \frac{|d t|}{\left|t-w_{1}\right|^{\left(2+\gamma_{1}\right)\left(2-\nu_{1}\right)}} \preceq n^{\left(2+\gamma_{1}\right)\left(2-\nu_{1}\right)-1} .
$$

1.2.2) For any $\gamma_{1}>-2$, according to Lemmas 2.1 and 2.2, we have:

$$
\begin{aligned}
D_{n, 1}^{2}(w) & \preceq \int_{K_{2,1}^{1}\left(R_{1}\right)} \frac{|d t|}{|\Psi(t)-\Psi(w)|^{2+\gamma_{1}}}+\int_{K_{2,2}^{1}\left(R_{1}\right)} \frac{|d t|}{\left|\Psi(t)-\Psi\left(w_{1}\right)\right|^{2+\gamma_{1}}} . \\
& \preceq \int_{K_{2,1}^{1}\left(R_{1}\right)} \frac{|d t|}{|t-w|^{\left(2+\gamma_{1}\right) 1+\varepsilon}}+1 \preceq n^{\left(2+\gamma_{1}\right)(1+\varepsilon)-1}, \forall \varepsilon>0 .
\end{aligned}
$$

Combining estimates (3.14)-(3.26), for $w \in \Phi\left(L_{R}\right)$, we have:

$$
D_{n, 1} \preceq n^{\left(2+\widetilde{\gamma}_{1}\right)\left(2-\nu_{1}\right)-1+\varepsilon}, \widetilde{\gamma}_{1}:=\max \left\{0 ; \gamma_{1}\right\} .
$$

Case 2. Let $w \in \Phi\left(L_{R}^{2}\right)$. Analogously to the Case 1, we will obtain estimates for $w \in K_{1}^{2}(R)$ and $w \in K_{2}^{2}(R)$

$$
D_{n, 2}(w) \preceq n^{\left(2+\gamma_{2}\right)\left(2-\nu_{2}\right)-1+\varepsilon}, \widetilde{\gamma}_{2}:=\max \left\{0 ; \gamma_{2}\right\}
$$

Therefore, comparing relations (3.11), (3.13), (3.27) and (3.28), we have:

$$
D_{n}(w) \preceq n^{\left(2+\widetilde{\gamma}_{1}\right)\left(2-\nu_{1}\right)-1}+n^{\left(2+\widetilde{\gamma}_{2}\right)\left(2-\nu_{2}\right)-1},
$$

where $\widetilde{\gamma}_{1}$ and $\widetilde{\gamma}_{2}$ defined as in (3.27) and (3.28).

Now, from (3.7), (3.8), (3.9) and (3.29), for any $z \in L_{R}$, we get:

$$
\left|P_{n}(z)\right| \preceq\left[n^{\left(2+\widetilde{\gamma}_{1}\right)\left(2-\nu_{1}\right)}+n^{\left(2+\widetilde{\gamma}_{2}\right)\left(2-\nu_{2}\right)}\right]\left\|P_{n}\right\|_{p}
$$

Since this estimate holds for any $z \in L_{R}$, then it is also true for $z \in \bar{G}$. Therefore, we complete the proof of theorem.

\subsection{Proof of Theorem 1.2}

Proof. Suppose that $G \in P A C\left(\nu_{1}, \nu_{2}\right)$ for some $0<\nu_{1}, \nu_{2}<1$ and $h(z)$ is defined as in (1.1). For each $R>1$, let $w=\varphi_{R}(z)$ denote a univalent conformal mapping $G_{R}$ onto the $B$, normalized by $\varphi_{R}(0)=0, \varphi_{R}^{\prime}(0)>0$, and let $\left\{\zeta_{j}\right\}, 1 \leq j \leq m \leq n$, be a zeros of $P_{n}(z)$ (if any exist) lying on $G_{R}$. Let

$$
b_{m, R}(z):=\prod_{j=1}^{m} \widetilde{b}_{j, R}(z)=\prod_{j=1}^{m} \frac{\varphi_{R}(z)-\varphi_{R}\left(\zeta_{j}\right)}{1-\overline{\varphi_{R}\left(\zeta_{j}\right)} \varphi_{R}(z)}
$$


denote a Blaschke function with respect to zeros $\left\{\zeta_{j}\right\}, 1 \leq j \leq m \leq n$, of $P_{n}(z)$ ([33]). Clearly,

$$
\left|b_{m, R}(z)\right| \equiv 1, z \in L_{R} \text {, and }\left|b_{m, R}(z)\right|<1, z \in G_{R} .
$$

For any $p>0$ and $z \in G_{R}$, let us set

$$
T_{n . p}(z):=\left[\frac{P_{n}(z)}{b_{m, R}(z)}\right]^{p / 2} .
$$

The function $T_{n, p}(z)$ is analytic in $G_{R}$, continuous on $\bar{G}_{R}$ and does not have zeros in $G_{R}$. We take an arbitrary continuous branch of the $T_{n, p}(z)$ and for this branch we maintain the same designation. Then, the Cauchy integral representation for the $T_{n, p}(z)$ at the $z=z_{1}$ gives:

$$
T_{n, p}\left(z_{1}\right)=\frac{1}{2 \pi i} \int_{L_{R}} T_{n, p}(\zeta) \frac{d \zeta}{\zeta-z_{1}} .
$$

Then, according to (3.31), we obtain:

$$
\begin{aligned}
\left|P_{n}\left(z_{1}\right)\right|^{p / 2} & \leq \frac{\left|b_{m, R}\left(z_{1}\right)\right|^{p / 2}}{2 \pi} \int_{L_{R}}\left|\frac{P_{n}(\zeta)}{b_{m, R}(\zeta)}\right|^{p / 2} \frac{|d \zeta|}{\left|\zeta-z_{1}\right|} \\
& \preceq \int_{L_{R}}\left|P_{n}(\zeta)\right|^{p / 2} \frac{|d \zeta|}{\left|\zeta-z_{1}\right|} .
\end{aligned}
$$

Multiplying the numerator and the denominator of the last integrand by $h^{1 / 2}(\zeta)$, replacing the variable $w=\Phi(z)$ and applying the Hölder inequality, we obtain:

$$
\begin{aligned}
& \left(\int_{L_{R}}\left|P_{n}(\zeta)\right|^{\frac{p}{2}} \frac{|d \zeta|}{\left|\zeta-z_{1}\right|}\right)^{2} \\
\leq & \int_{|t|=R} h(\Psi(t))\left|P_{n}(\Psi(t))\right|^{p}\left|\Psi^{\prime}(t)\right|^{2}|d t| \cdot \int_{|t|=R} \frac{|d t|}{h(\Psi(t))\left|\Psi(t)-\Psi\left(w_{1}\right)\right|^{2}} \\
= & \int_{|t|=R}\left|f_{n, p}(t)\right|^{p}|d t| \cdot \int_{|t|=R} \frac{|d t|}{h(\Psi(t))\left|\Psi(t)-\Psi\left(w_{1}\right)\right|^{2}},
\end{aligned}
$$

where $f_{n, p}(t)$ has been defined as in (3.8). Since $R>1$ is arbitrary, then (3.34) holds also for $R=R_{1}:=$ $1+\frac{\varepsilon_{1}}{n}, 0<\varepsilon_{1}<1$. So, we have:

$$
\begin{aligned}
& \left(\int_{L_{R_{1}}}\left|P_{n}(\zeta)\right|^{\frac{p}{2}} \frac{|d \zeta|}{\left|\zeta-z_{1}\right|}\right)^{2} \\
\leq & \left(\int_{|t|=R_{1}}\left|f_{n, p}(t)\right|^{p}|d t|\right) \cdot\left(\int_{|t|=R_{1}} \frac{|d t|}{h(\Psi(t))\left|\Psi(t)-\Psi\left(w_{1}\right)\right|^{2}}\right) \\
= & : A_{n} \cdot D_{n}\left(w_{1}\right),
\end{aligned}
$$

and, $A_{n}$ and $D_{n}\left(w_{j}\right)$ have been defined as in (3.8) for $R=R_{1}$. Therefore, from (3.33) and (3.35), we have:

$$
\left|P_{n}\left(z_{1}\right)\right| \preceq A_{n} \cdot D_{n}\left(w_{1}\right),
$$

where, according to (3.9), the estimate

$$
A_{n} \preceq n \cdot\left\|P_{n}\right\|_{p}^{p}
$$


is satisfied. For the estimate of the quantity $D_{n}\left(w_{1}\right)$ we use the notations at the estimation of the $D_{n}(w)$ as in (3.11)-(3.13). Therefore, under these notations, for the $D_{n}\left(w_{1}\right)$, we get:

$$
\begin{gathered}
D_{n}\left(w_{1}\right) \preceq \int_{\Phi\left(L_{R_{1}}^{j}\right)} \frac{|d t|}{\left|\Psi(t)-\Psi\left(w_{1}\right)\right|^{2+\gamma_{1}}} \\
\preceq \sum_{i=1}^{2} \int_{K_{i}^{1}\left(L_{R_{1}}\right)} \frac{|d t|}{\left|\Psi(t)-\Psi\left(w_{1}\right)\right|^{2+\gamma_{1}}}=: \sum_{i=1}^{2} D_{n, 1}^{i}\left(w_{1}\right) .
\end{gathered}
$$

So, we need to evaluate the $D_{n, 1}^{i}\left(w_{1}\right)$ for each $i=1,2$. We have:

$$
\begin{gathered}
D_{n, 1}^{1}\left(w_{1}\right)=\int_{K_{1}^{1}\left(L_{R_{1}}\right)} \frac{|d t|}{\left|\Psi(t)-\Psi\left(w_{1}\right)\right|^{2+\gamma_{1}}} \\
\preceq \int_{K_{1}^{1}\left(L_{R_{1}}\right)} \frac{|d t|}{\left|t-w_{1}\right|^{\left(2+\gamma_{1}\right)\left(2-\nu_{1}\right)}} \preceq\left\{\begin{array}{cl}
n^{\left(2+\gamma_{1}\right)\left(2-\nu_{1}\right)-1}, & \text { if }\left(2+\gamma_{1}\right)\left(2-\nu_{1}\right)>1, \\
\ln n, & \text { if }\left(2+\gamma_{1}\right)\left(2-\nu_{1}\right)=1, \\
1, & \text { if }\left(2+\gamma_{1}\right)\left(2-\nu_{1}\right)<1,
\end{array}\right.
\end{gathered}
$$

and

$$
D_{n, 1}^{2}\left(w_{1}\right)=\int_{K_{1}^{2}\left(L_{R_{1}}\right)} \frac{|d t|}{\left|\Psi(t)-\Psi\left(w_{1}\right)\right|^{2+\gamma_{1}}} \preceq \int_{K_{1}^{2}\left(L_{R_{1}}\right)} \frac{|d t|}{\left|t-w_{1}\right|^{2+\gamma_{1}+\varepsilon}} \preceq n^{\left(2+\gamma_{1}\right)(1+\varepsilon)-1} .
$$

Combining relations (3.37) - (3.39), we have:

$$
D_{n}\left(w_{1}\right) \preceq\left\{\begin{array}{cl}
n^{\left(2+\gamma_{1}\right)\left(2-\nu_{1}\right)-1+\varepsilon}, & \text { if }\left(2+\gamma_{1}\right)\left(2-\nu_{1}\right)>1-\varepsilon, \\
\ln n, & \text { if }\left(2+\gamma_{1}\right)\left(2-\nu_{1}\right)=1-\varepsilon, \\
1, & \text { if }\left(2+\gamma_{1}\right)\left(2-\nu_{1}\right)<1-\varepsilon,
\end{array}\right.
$$

From the estimations (3.36) and (3.40), we obtain:

$$
\left|P_{n}\left(z_{1}\right)\right| \preceq\left\{\begin{array}{cl}
n^{\frac{\left(2+\gamma_{1}\right)\left(2-\nu_{1}\right)}{p}+\varepsilon}, & \text { if }\left(2+\gamma_{1}\right)\left(2-\nu_{1}\right)>1-\varepsilon, \\
(n \ln n)^{\frac{1}{p}}, & \text { if }\left(2+\gamma_{1}\right)\left(2-\nu_{1}\right)=1-\varepsilon,\left\|P_{n}\right\|_{p}, \\
n^{\frac{1}{p}}, & \text { if }\left(2+\gamma_{1}\right)\left(2-\nu_{1}\right)<1-\varepsilon,
\end{array}\right.
$$

and we complete the proof of theorem.

Acknowledgments. This work is supported by KTMU Project No: 2016 FBE 13.

\section{References}

1. F.G. Abdullayev, V.V. Andrievskii, On the orthogonal polynomials in the domains with $K$-quasiconformal boundary. Izv. Akad. Nauk Azerb. SSR., Ser. FTM, no.1, pp.3-7, 1983.

2. F.G. Abdullayev, On the some properties of the orthogonal polynomials over the region of the complex plane (Part I), Ukr.Math.J., vol. 52, no.12, pp. 1807-1821, 2000.

3. F.G. Abdullayev, On the some properties of the orthogonal polynomials over the region of the complex plane (Part II), Ukr.Math.J., vol. 53, no. 1, pp. 1-14, 2001.

4. F.G. Abdullayev, On the some properties of the orthogonal polynomials over the region of the complex plane (Part III), Ukr.Math.J., vol. 53, no. 12, pp. 1934-1948, 2001.

5. F.G. Abdullayev, The properties of the orthogonal polynomials with weight having singulerity on the boundary contour, J. of Comp. Anal. and Appl., vol. 6, no. 1, pp. 43-59, 2004,.

6. F.G. Abdullayev, U. Deger, On the orthogonal polynomials with weight having singularity on the boundary of regions of the complex plane, Bull. Belg. Math. Soc., vol. 16, no. 2, pp. 235-250, 2009. 
7. F. G. Abdullayev, C.D. Gün, On the behavior of the algebraic polynomials in regions with piecewise smooth boundary without cusps, Ann.Polon.Math., vol. 111, pp. 39-58, 2014.

8. F. G. Abdullayev, N.P. Özkartepe, On the Behavior of the Algebraic Polynomial in Unbounded Regions with Piecewise Dinı-Smooth Boundary, Ukr.Math.J., vol. 66, no. 5, pp. 579-597, 2014.

9. F. G. Abdullayev, N.P. Özkartepe, Uniform and pointwise Bernstein-Walsh-type inequalities on a quasidisk in the complex plane, Bull. Belg. Math. Soc., vol. 23, no. 2, pp. 285-310, 2016.

10. F.G. Abdullayev, T. Tunç, Uniform and pointwise polynomial inequalities in regions with asymptotically conformal curve on weighted Bergman space, Lobachevcki Journal of Mathematics, vol. 38, no. 2, pp. 193-205, 2017.

11. F.G. Abdullayev, T. Tunç, G. A. Abdullayev, Polynomial inequalities in quasidisks on weighted Bergman space, Ukr. Math. J., vol. 69, no. 5, pp. 675-695, 2017.

12. F.G. Abdullayev, D. Şimşek, N. Saypidinova and Z. Tashpaeva, Polynomial Inequalities in Regions with Piecewise Asymptotically Conformal Curve in the Weighted Lebesgue Space, Advances in Analysis, vol. 3, no. 2, pp. 100-112. 2018.

13. L. Ahlfors, Lectures on Quasiconformal Mappings. Princeton, NJ: Van Nostrand, 1966.

14. J. M.Anderson, J. Becker, F. D. Lesley, Boundary values of asymptotically conformal mapping, J. London Math. Soc., vol. 38, no. 2, pp. 453-462, 1988.

15. V. V. Andrievskii, Weighted Polynomial Inequalities in the Complex Plan, J. Approx.Theory, vol. 164, no. 9, pp. 1165-1183, 2012.

16. S. Balci, M. Imash-kyzy, F.G. Abdullayev, Polynomial Inequalities in Regions with Zero Interior Angles in the Bergman Space, Ukr.Math.J., vol. 70, no. 3, pp. 362-384, 2018.

17. I.M. Batchayev, Integral representations in domains with quasiconformal boundary and some of their applications. Avtoreferat dis. cand.fiz.-mat. nauk Azerb. Univ., Baku, 16 p. 1981.

18. P.P. Belinskii, General Properties of Quasiconformal Mappings. Nauka, Sib. otd., Novosibirsk, 1974.

19. J. Becker, C. Pommerenke, Uber die quasikonforme Fortsetzung schlichten Funktionen, Math. Z., vol. 161, pp. 69-80, 1978.

20. E.M. Dyn'kin, Nonanalytic symmetry principle and conformal mappings. St. Petersburg Math. J., no. 5, pp. 523-544, 1994.

21. V. Gutlyanskii, V. Ryazanov, On asymptotically conformal curves, Complex Variables, vol. 25, pp. 357-366, 1994.

22. V. Gutlyanskii, V. Ryazanov, On the local behaviour of quasi-conformal mappings, Izvestiya: Mathematics, vol. 59, no. 3, pp. 471-498, 1995.

23. V. Ya. Gutlyanskii, V. I. Ryazanov, On quasi-circles and asymptotically conformal circles, Dokl. Ross. Akad. Nauk, vol. 330, no. 5, pp. 546-548, 1993. (English transl., Russian Acad. Sci. Math., vol. 47, pp. 563-566, 1993).

24. O. Lehto, K.I. Virtanen, Quasiconformal Mapping in the plane, Springer Verlag, Berlin, 1973.

25. F.D. Lesley, Hölder continuity of conformal mappings at the boundary via the strip method, Indiana Univ. Math. J., vol. 31, pp. 341-354, 1982.

26. G. V. Milovanovic, D.S. Mitrinovic, Th.M. Rassias, Topics in Polynomials: Extremal Problems, Inequalities, Zeros, Singapore, World Scientific, 1994.

27. S. M. Nikolskii, Approximation of Function of Several Variable and Imbeding Theorems, New-York, SpringerVerlag, 1975.

28. S. Rickman, Characterization of quasiconformal arcs, Ann. Acad. Sci. Fenn., Ser. A, Mathematica., vol. 395, no. 30,1966 .

29. Ch. Pommerenke, Univalent Functions, Göttingen, Vandenhoeck \& Ruprecht, 1975.

30. Ch.Pommerenke, Boundary Behaviour of Conformal Maps. - Springer-Verlag, Berlin, 1992.

31. Ch. Pommerenke, S.E.Warschawski, On the quantitative boundary behavior of conformal maps, Comment. Math. Helv., vol. 57, pp. 107-129, 1982.

32. I. Pritsker, Comparing Norms of Polynomials in One and Several Variables, J. of Math. Anal. and Appl., no. 216, pp. 685-695, 1997.

33. J.L. Walsh, Interpolation and Approximation by Rational Functions in the Complex Domain, AMS, 1960.

34. S.E. Warschawski, Über das randverhalten der ableitung der ableitung der abbildungsfunktion bei konformer abbildung, Math.Z., vol. 35, pp. 321-456, 1932. 